\title{
Immune Checkpoint Inhibitor-associated New-onset Primary Adrenal Insufficiency: A Retrospective Analysis Using the FAERS
}

Difei Lu

Peking University First Hospital

Jun Yao

Peking University First Hospital

Geheng Yuan

Peking University First Hospital

Ying Gao ( $\square$ bjgaoying@yahoo.com )

Peking University First Hospital https://orcid.org/0000-0003-3953-8900

Junqing Zhang

Peking University First Hospital

Xiaohui Guo

Peking University First Hospital

\section{Research Article}

Keywords: primary adrenal insufficiency, immune checkpoint inhibitors, immune-related adverse event, prognosis

Posted Date: October 4th, 2021

DOI: https://doi.org/10.21203/rs.3.rs-702598/v2

License: (c) (i) This work is licensed under a Creative Commons Attribution 4.0 International License. Read Full License 


\section{Abstract}

\section{Background}

Our study aimed to investigate the prevalence and demographic characteristics of immune checkpoint inhibitor-associated primary adrenal insufficiency (ICI-PAI), and to explore the risk factors of clinical outcome using data from the US FDA Adverse Event Reporting System (FAERS).

\section{Methods}

This is a retrospective study. All cases of new-onset or newly-diagnosed primary adrenal insufficiency associated with FDA approved ICls from $1^{\text {st }}$ January 2007 to $31^{\text {st }}$ December 2020 were identified and collected using FAERS. Data of age, sex category, body weight of participated individuals, the reporting year and the prognosis of cases, and other accompanied endocrinopathies related to ICls were analyzed.

Results

The incidence of ICl-PAI was $1.03 \%(1180 / 114121)$. The application of the combination therapy of antiCTLA-4 and anti-PD-1 was related to higher risk of PAI compared with anti-PD-1 group $\left(\chi^{2}=92.88\right.$, $p<0.001$ ). Male and elder individuals showed higher risk of ICI-PAI (male vs. female, $1.17 \%$ vs. $0.94 \%$, $\chi^{2}=12.55, p<0.001$; age $<65$ vs. $\geq 65,1.20 \%$ vs. $\left.1.41 \%, \chi^{2}=6.89, p=0.009\right)$. The co-occurrence rate of other endocrinopathies aside from PAI was $24.3 \%$, which showed a higher trend in patients on nivolumabipilimumab treatment compared with those on PD-1 inhibitors $\left(\chi^{2}=3.227, p=0.072\right)$. Body weight was negatively associated with the risk of death in the study population [ $p=0.033$ for the regression model; $\mathrm{B}=-0.017$, OR 0.984, 95\% Cl (0.969-0.998), $p=0.029]$.

\section{Conclusions}

ICl-associated PAI is a rare but important irAE. Male and elder patients have higher risk of ICI-PAI. Awareness among clinicians is critical when patients with lower body weight develop PAl, which indicates poor clinical outcomes.

\section{Background}

Immune checkpoint inhibitors (ICls), including programmed cell death protein-1 (PD-1) inhibitors, programmed death-ligand 1 (PD-L1) inhibitors and cytotoxic T cell-associated protein-4 (CTLA-4) inhibitors, have demonstrated potent and prolonged antitumor activity in patients with advanced malignancy during the last decade. Immune-related adverse events (irAEs) of ICls, including endocrinopathies, were recognized in a subgroup of patients receiving $\mathrm{ICI}$ treatment [1]. Primary adrenal insufficiency (PAI) was a type of ICl-related endocrinopathy, second in line to the prevalence of thyroid dysfunction and hypophysitis, while preceding type 1 diabetes mellitus (T1DM) [2]. 
ICls-associated PAI (ICl-PAI) was first reported on Dec 21, 2007 in a 56-year-old female patient receiving ipilimumab mono-therapy in the data from the US FDA Adverse Event Reporting System (FAERS). In clinical practice, patients with advanced stage of cancer could develop adrenal insufficiency by ICl-related PAI, ICl-related hypophysitis, or bilateral adrenal metastasis [3]. ICI-PAI could be distinguished from secondary adrenal insufficiency caused by ICl-related hypophysitis owing to the complete destruction of adrenal glands, including the zona glomerulosa producing mineralocorticoid. As a potentially lifethreatening subtype of endocrine-related irAEs that can lead to adrenal crisis and death, it is critical for both endocrinologists and oncologists to obtain accurate data from large population of the incidence and clinical manifestations of ICI-PAI. However, the incidence of ICI-PAI was only described in a few case reports and varied among statistics [5-7]. We carried on a retrospective study to investigate the demographic characteristics of ICI-PAI, and to explore risk factors of clinical outcome using data from the FAERS.

\section{Methods}

Our study is a retrospective study based on the FAERS database, mainly focused on ICI-related PAI. The FAERS is a public-open database for adverse events induced by all FDA approved medications. All cases of new-onset or new-diagnosed primary adrenal insufficiency associated with FDA approved ICls from 1st January 2007 to 31 st December 2020 were identified and accumulated in our study population using FAERS. The ICI medications included in this study were anti-CTLA-4 mono-antibody (ipilimumab), anti-PD1 mono-antibodies (nivolumab, pembrolizumab and cemiplimab) and anti-PD-L1 mono-antibodies (atezolizumab, avelumab and durvalumab).

New-onset PAI or adrenalitis in patients receiving ICI treatment was considered the diagnosis of ICI-PAI. The definition of ICI-PAI included the following FAERS terms as adverse reaction: adrenal insufficiency, adrenalitis, and primary adrenal insufficiency. Those with secondary adrenal insufficiency, pituitary adrenal insufficiency or hypophysitis after ICls treatment, according to the reported adverse reactions, were excluded from the study population. When there was uncertainty in the origin of adrenal insufficiency, that is to say, cases diagnosed with primary adrenal insufficiency and hypophysitis or secondary/pituitary adrenal insufficiency at the same time, were excluded from our study. Data of age, sex categories, the reporting year of cases, the body weight of participated individuals, the other accompanied endocrinopathies related to ICls and prognosis was collected. The co-occurrence of a second immune-related endocrinopathy besides PAI was defined as the situation of other accompanied endocrinopathies. In FAERS, patients labeled on the outcomes of hospitalization, disabled, life-threatened and death, were considered as severe cases, otherwise they were assigned to non-serious cases. Since all cases in the study were anonymous and all data was available in public resource, the study was exempt from informed consent and ethics.

Statistics 
Quantitative variables in accordance with normal distribution were analyzed using one-way ANOVA test. The proportion of ICl-PAI in total ICls related adverse events during the same study period or population were quantitative variables, and were compared using $\chi^{2}$ test by sex categories, age and reporting year after Bonferroni adjustment method. We used logistic regression analysis to evaluate the risk of poor prognosis adjusting confounders including sex, age, body weight and subtypes of polyendocrinopathies in patients receiving different regimens. Statistical analysis was performed using SPSS 17.0 (IBM, U.S.). $\mathrm{P}<0.05$ was considered statistical significance.

\section{Results}

\section{Incidences of ICI-PAI in all cases with adverse events}

In total, 1180 cases of ICI-PAI were identified in 114121 patients reported as adverse events related to ICls, and the incidence of ICI-PAI was $1.03 \%$. In patients on PD- 1 inhibitors, the incidence of PAI was $0.77 \%$ (578/74605), $0.60 \%$ (97/16071) in those on PD-L1 inhibitors, $0.68 \%$ (160/23445) in patients on CTLA-4 inhibitors and $1.47 \%$ (345/23445) in those on the combination therapy of ipilimumab and nivolumab. Patients on PD-1 inhibitors had higher risk of PAl compared with anti-PD-L1 group $\left(\chi^{2}=5.14, p=0.022\right)$, and showed significant lower risk of PAl comparing with the combination therapy group $\left(\chi^{2}=92.88, p<\right.$ 0.001). There was no reported adrenal crisis in FAERS.

\section{Demographic characteristics of ICls-PAI}

Overall, 738 individuals (62.5\%) were male, 359 (30.4\%) were female and 83 subjects $(7.1 \%)$ were not specified. Male individuals showed higher risk of ICI-PAI [male vs. female, $1.17 \%$ (738/62594) vs. $0.94 \%$ (359/38179), $\left.\chi^{2}=12.55, p<0.001\right]$, and the significant predisposition of men was still observed in those receiving anti-PD-1 therapy and the combination therapy of anti-PD-1 and anti-CTLA-4 [anti-PD-1 therapy, male vs. female, $0.93 \%$ (384/41282) vs. $0.58 \%$ (144/24673), $\chi^{2}=23.36, p<0.001$; anti-PD-L1 therapy, $0.63 \%(54 / 8522)$ vs. $0.57 \%(35 / 6099), \chi^{2}=0.21, p=0.647$; anti-CTLA-4 therapy, $0.74 \%(95 / 12790)$ vs. $0.76 \%(56 / 7407), \chi^{2}=0.01, p=0.916$; anti-PD- $1+$ anti-CTLA-4 therapy, $1.60 \%(205 / 12790)$ vs. $1.67 \%$ (124/7407), $\left.\chi^{2}=0.09, p=0.761\right]$.

In the aspect of age distribution, 439 (37.2\%) patients were less than 65 year-olds, $588(49.8 \%)$ were equal to or above 65 year-olds, and $153(13.0 \%)$ were not specified. The incidence of ICls-PAI was significantly reduced in younger patients [age < 65 years, $439 / 36672(1.20 \%)$ ] comparing with those elder than 65 year-olds [588/41679 $\left.(1.41 \%), \chi^{2}=6.89, p=0.009\right]$. Similar results between different age groups were observed in patients receiving anti-PD- 1 therapy $[<65$ vs. $\geq 65,0.76 \%(171 / 22423)$ vs. $1.20 \%$ (330/27546), $\left.\chi^{2}=23.61, p<0.001\right]$ and the combined therapy of anti-PD-1 and anti-CTLA-4 [< 65 vs. $\geq 65$, $1.68 \%(142 / 8456)$ vs. $\left.2.35 \%(169 / 7194), \chi^{2}=0.8 .96, p=0.003\right]$.

\section{The co-occurrence other endocrinopathies in patients with ICls-PAI}


The co-occurrence rate of other endocrinopathies aside from PAI was $24.2 \%(286 / 1180)$ in all patients receiving $\mathrm{ICl}$ therapy. The most common type of accompanied endocrinopathy was thyroid dysfunction (166, $14.1 \%)$, followed by ICls-related type 1 diabetes (43/1180, 3.6\%). Among different regimens, the highest occurrence rate of accompanied endocrinopathy was $28.1 \%(97 / 345)$ in patients on nivolumabipilimumab treatment, followed by $23.8 \%(38 / 160)$ in patients on CTLA-4 inhibitors and $22.8 \%(132 / 578)$ in those on PD-1 inhibitors, and the lowest rate was 19\% (19/97) in those on PD-L1 inhibitors. There was no significant difference of other ICl-associated endocrinopathies between patients on anti-CTLA-4 therapy and on the combination therapy of nivolumab and ipilimumab $\left(X^{2}=1.064, p=0.302\right)$. An increase trend was discovered in patients on the combination therapy compared with those on PD- 1 inhibitors $\left(\chi^{2}\right.$ $=3.227, p=0.072$ ). Also, no significant difference was found in the occurrence rate of other ICl-associated endocrinopathies between patients on anti-PD-L1 therapy and on anti-PD-1 therapy $\left(X^{2}=0.043, p=0.836\right)$.

We further analyzed the subtypes of accompanied ICI-related endocrine diseases. In ICls-PAI patients accompanied with T1DM, only one patient with PAI on anti-CTLA-4 mono-therapy developed T1DM (1/160). No significant difference was discovered in the incidence of T1DM in PAI patients on PD-1 inhibitors and nivolumab-ipilimumab treatment $\left(X^{2}=2.995, p=0.084\right)$. Also, no difference of the distribution of accompanying thyroid dysfunction was found among the four regimens $\left(\chi^{2}=1.860, p=\right.$ 0.602).

\section{Distribution of ICls-PAI based on reporting year}

Overall, the proportion of ICI-PAI to all reported ICls related adverse events significantly increased from $0.67 \%(71 / 10590)$ in 2016 to $1.25 \%(378 / 30246)$ in $2020\left(\chi^{2}=24.21, p<0.001\right.$, Bonferroni corrected, Table 1). In anti-PD1 therapy subgroup, the incidence of PAl significantly raised from $0.48 \%(18 / 3788)$ in 2015 and before to $0.98 \%(188 / 19254)$ in $2020\left(X^{2}=8.98, p=0.003\right.$, Bonferroni corrected). The incidence of PAl in subjects on anti-PD-L1 therapy remained 0.90\% (15/1662) in 2017 to $0.64 \%(40 / 6221)$ in 2020 $\left(X^{2}=1.27, p=0.259\right.$, Bonferroni corrected). The reported number of ICI-PAl in patients on anti-CTLA-4 mono-therapy declined over time, from 1.50\% (93/6200) in 2015 and before to $0.06 \%$ (3/4771) in 2020 (Fisher's exact test, $p<0.001$, Bonferroni corrected). A significant increase was discovered in incidence of PAl in individuals receiving the combined regimen of anti-PD- 1 and anti-CTLA- 4 therapy, from $0.42 \%$ $(26 / 6200)$ in 2015 and before to $3.08 \%(147 / 4771)$ in 2020 ( $\chi^{2}=123.08, p<0.001$, Bonferroni corrected). 
Table 1

Clinical characteristics of patients with ICl-related primary adrenal insufficiency

\begin{tabular}{|c|c|c|c|c|c|}
\hline Characteristics & $\begin{array}{l}\text { All } \\
(n= \\
1180)\end{array}$ & $\begin{array}{l}\text { Anti-PD-1 } \\
\text { therapy } \\
(n=578)\end{array}$ & $\begin{array}{l}\text { Anti-PD-L1 } \\
\text { therapy } \\
(n=97)\end{array}$ & $\begin{array}{l}\text { Anti-CTLA-4 } \\
\text { therapy } \\
(n=160)\end{array}$ & $\begin{array}{l}\text { Anti-PD- } 1 \text { and anti- } \\
\text { CTLA-4 therapy } \\
(n=345)\end{array}$ \\
\hline \multicolumn{6}{|c|}{ Sex categories $(n, \%)$} \\
\hline Male & $\begin{array}{l}738 \\
(62.5)\end{array}$ & $384(66.4)$ & $54(55.7)$ & $95(59.4)$ & $205(59.4)$ \\
\hline Female & $\begin{array}{l}359 \\
(30.4)\end{array}$ & $144(24.9)$ & $35(36.1)$ & $56(35.0)$ & $124(35.9)$ \\
\hline Not specified & $\begin{array}{l}83 \\
(7.1)\end{array}$ & $50(8.7)$ & $8(8.2)$ & $9(5.6)$ & $16(4.6)$ \\
\hline \multicolumn{6}{|l|}{ Age (years) $(n, \%)$} \\
\hline $18-64$ & $\begin{array}{l}439 \\
(37.2)\end{array}$ & $171(29.6)$ & $41(43.3)$ & $85(53.1)$ & $142(41.2)$ \\
\hline$\geq 65$ & $\begin{array}{l}588 \\
(49.8)\end{array}$ & $330(57.1)$ & $39(40.2)$ & $50(31.3)$ & $169(49.0)$ \\
\hline Not specified & $\begin{array}{l}153 \\
(13.0)\end{array}$ & 77 (13.3) & $17(17.5)$ & $25(15.6)$ & $34(9.9)$ \\
\hline \multicolumn{6}{|c|}{ Reporting year (n, \%) } \\
\hline 2015 and before & $\begin{array}{l}134 \\
(11.4)\end{array}$ & $18(3.1)$ & $0(0)$ & $93(58.1)$ & $26(7.5)$ \\
\hline 2016 & $\begin{array}{l}71 \\
(6.0)\end{array}$ & $36(6.2)$ & $0(0)$ & $16(10.0)$ & $19(5.5)$ \\
\hline 2017 & $\begin{array}{l}139 \\
(11.8)\end{array}$ & $78(13.5)$ & $15(15.5)$ & $17(10.6)$ & $29(8.4)$ \\
\hline 2018 & $\begin{array}{l}186 \\
(15.8)\end{array}$ & $97(16.8)$ & $22(22.7)$ & $21(13.1)$ & $46(13.3)$ \\
\hline 2019 & $\begin{array}{l}276 \\
(23.4)\end{array}$ & $160(27.7)$ & $20(20.6)$ & $10(6.3)$ & $86(24.9)$ \\
\hline 2020 & $\begin{array}{l}378 \\
(32.0)\end{array}$ & $188(32.5)$ & $40(41.2)$ & $3(1.9)$ & $147(42.6)$ \\
\hline \multicolumn{6}{|c|}{ Accompanied with other endocrinologic irAEs (n, \%) } \\
\hline Total & $\begin{array}{l}286 \\
(24.2)\end{array}$ & $132(22.8)$ & $19(19.6)$ & $38(23.8)$ & $97(28.1)$ \\
\hline
\end{tabular}

* Accompanied endocrinopathies besides PAI, T1DM or thyroid dysfunction. 


\begin{tabular}{|c|c|c|c|c|c|}
\hline Characteristics & $\begin{array}{l}\text { All } \\
(n= \\
1180)\end{array}$ & $\begin{array}{l}\text { Anti-PD-1 } \\
\text { therapy } \\
(n=578)\end{array}$ & $\begin{array}{l}\text { Anti-PD-L1 } \\
\text { therapy } \\
(n=97)\end{array}$ & $\begin{array}{l}\text { Anti-CTLA-4 } \\
\text { therapy } \\
(n=160)\end{array}$ & $\begin{array}{l}\text { Anti-PD-1 and anti- } \\
\text { CTLA-4 therapy } \\
(n=345)\end{array}$ \\
\hline T1DM & $\begin{array}{l}43 \\
(3.6)\end{array}$ & $21(3.6)$ & $0(0)$ & $1(0.6)$ & $21(6.1)$ \\
\hline $\begin{array}{l}\text { Thyroid } \\
\text { dysfunction }\end{array}$ & $\begin{array}{l}166 \\
(14.1)\end{array}$ & $89(15.4)$ & $12(12.4)$ & $23(14.4)$ & $42(12.2)$ \\
\hline $\begin{array}{l}\text { Other } \\
\text { endocrinopathies } \\
\star\end{array}$ & $\begin{array}{l}77 \\
(6.5)\end{array}$ & $22(3.8)$ & $7(7.2)$ & $14(8.8)$ & $34(9.9)$ \\
\hline \multicolumn{6}{|l|}{ Prognosis (n, \%) } \\
\hline Non-serious & $\begin{array}{l}243 \\
(20.6)\end{array}$ & $122(21.1)$ & $27(27.8)$ & $45(28.1)$ & $49(14.2)$ \\
\hline Hospitalized & $\begin{array}{l}631 \\
(53.5)\end{array}$ & $290(50.2)$ & $51(52.6)$ & $88(55.0)$ & $202(58.6)$ \\
\hline $\begin{array}{l}\text { Life-threatened or } \\
\text { disabled }\end{array}$ & $\begin{array}{l}165 \\
(14.0)\end{array}$ & $92(15.9)$ & $6(6.2)$ & $10(6.3)$ & $57(16.5)$ \\
\hline Death & $\begin{array}{l}141 \\
(11.9)\end{array}$ & $74(12.8)$ & $13(13.4)$ & 17 (10.6) & $37(10.7)$ \\
\hline
\end{tabular}

In terms of prognosis, 937 (79.4\%) cases were severe and 243 (20.6\%) were non-serious. Among the severe cases, 631 (53.5\%) of them were hospitalized, 165 (14.0\%) were life-threatened or disabled, and $141(11.9 \%)$ cases of death due to various causes. The risk of severe AE increased in patients on the combination therapy of anti-PD-1 and anti-CTLA-4 compared with those on PD- 1 inhibitors [OR 1.616, $95 \% \mathrm{Cl}(1.125-2.322), p=0.009]$. A tendency of lowered risk of severe AE was observed in patients on CTLA-4 inhibitors compared with those on PD-1 inhibitors $\left(\chi^{2}=3.53, p=0.060\right)$, while the occurrence of severe $A E$ was insignificant in patients on the other different mono-therapy regimens (anti-PD- 1 vs. anti$\mathrm{PD}-\mathrm{L} 1, \chi^{2}=2.19, p=0.139$ ). Patients with PAI on anti-PD-1 therapy showed similar risk of death from all possible causes, including advanced malignancy, comparing with other regimens (anti-PD-L1, $\chi^{2}=0.03, p$ $=0.871$; anti-CTLA-4, $\chi^{2}=0.55, p=0.458$; anti-PD-1 + anti-CTLA-4, $\chi^{2}=0.88, p=0.348$ ).

Further analysis was conducted to explore confounders of the prognosis, including age, gender, other endocrinopathies and body weight, in patients on different regimens. Body weight was negatively associated with the risk of death in the study population $[p=0.033$ for the regression model; $\mathrm{B}=-0.017$, OR $0.984,95 \% \mathrm{Cl}(0.969-0.998), p=0.029]$, while different regimens, sex, age and endocrine comorbidities showed insignificant influence on the regression model. Similarly, increased body weight could reduce the risk of death in patients on the combination therapy of anti-PD- 1 and anti-CTLA-4 [ $p=0.031$ for the 
regression model; $\mathrm{B}=-0.041, \mathrm{OR} 0.960,95 \% \mathrm{Cl}(0.932-0.989), p=0.007]$. In subgroup of $\mathrm{PD}-1$ inhibitors, the comorbidity of ICl-related thyroid dysfunction (ICI-TD) was positively related to the risk of severe clinical prognosis $[p=0.049$ for the regression model; $\mathrm{B}=1.225$, OR $3.508,95 \% \mathrm{Cl}(1.074-11.458), p=$ 0.038].

\section{Discussion}

To our knowledge, this study represents the most recent and largest clinical description and characteristics of ICl-associated PAI using individual adverse events reports from FDA FAERS database. Although PAI is a rare adverse event, it calls for emergent reorganization and treatment for its possibility of adrenal crisis and death. Our study revealed the increasing incidence of ICl-associated PAI from 2016 to 2020. The increasing trend of incidence of ICI-PAI along with the reporting year may be explained by a more prevalent application of ICls therapy, also a more profound understanding of the disease by clinicians.

In our study, the incidence of ICI-PAI was 1.03\% (1180 to 114121) in all reported cases of ICI-related adverse events, and between $0.60 \%$ and $0.77 \%$ in individuals on mono-therapy, which was in accordance with the number of less than $1 \%$ in previous study [2]. Our research indicated an incidence of ICI-PAI of $1.47 \%$ in patients on the combination therapy of ipilimumab and nivolumab. This was lower than the number reported in patients on combination therapy, which was raised to $4 \%-8 \%$ [8]. In randomized clinical trials and meta-analysis, the incidence of ICI-PAI was up to $2.43 \%$ [9]. Since a proportion of nonserious cases may be not reported in the real-world practice, it may result in lowered incidence of ICI-PAI in FAERS database.

Our study suggested that ICI-PAI had a distinct gender and age distribution compared with other irAE endocrinopathies or autoimmune PAI. Of all 1180 cases of ICI-PAI, $62.5 \%$ were male in our study population. Similar result was concluded in a study using data from VigiBase, the World Health Organization's pharmacovigilance database, in which $54.4 \%$ of all 451 cases of PAl-irAE occurred in men [10]. However, studies revealed a female predominance on ICl-induced endocrinopathies [11], and different endocrinologic irAEs present with diverse gender predominance. A population study using data from FAERS consistently indicated that female had significant higher reporting frequencies in both hypothyroidism and hyperthyroidism, while hypophysitis showed a male dominated disparity [12].

Our study found a significant higher susceptibility of ICI-PAI in patients elder than 65 year-olds, and this predominance of elder patients was seen in individuals receiving anti-PD-1 therapy or the combined therapy of anti-PD- 1 and anti-CTLA-4. There was no report of patient under the age of 18 who developed ICl-induced PAI in FAERS, since the prevalence of lung cancer, renal cell carcinoma or melanoma was rare in under-age population.

Regarding the co-occurrence endocrinopathies, $24.2 \%$ of cases with ICI-PAI developed polyendocrinopathies. Ipilimumab mono-therapy or the combined therapy of anti-CTLA-4 and anti-PD-1 resulted in highest risk of polyendocrinopathies including PAI, followed by anti-PD-1 mono-therapy, which 
was consistent with other researchers [13]. This could be explicated by the possible destructive potential of ipilimumab and its combination therapy to endocrine glands, including thyroid glands and pancreas $\beta$ cells. More frequent surveillance on thyroid and adrenal function should be implemented in patients on CTLA-4 inhibitors or its combination therapy.

In our study, $79.4 \%$ of ICI-PAI cases were severe, and the mortality rate was $11.9 \%$ due to various causes. Previous study reported a severe adverse event rate of $91.1 \%$ and a mortality rate of $7.3 \%$ in PAl-irAE patients using VigiBase, in which the severe adverse event had a broadened definition of the outcome of death, being life-threatening, hospitalization, disability or any other medically important conditions [10]. In both studies, ICI-PAI could lead to a high proportion of serious outcome, which could be explained by the damage to adrenal gland and acute insufficiency of serum cortisol. Our study suggested that the risk of serious events increased in subgroup of the combination therapy of anti-PD- 1 and anti-CTLA-4, the risk of death or severe clinical outcome was insignificant in different mono-therapies. Some researchers concluded that patients on PD-1 inhibitors had raised mortality among ICls mono-therapy [14], and combination therapy of ICls was proved to be a positive factor of poor clinical outcome [15-16].

In our study, further analysis of prognostic factors suggested that lower body weight could lead to higher risk of death in the study population. Cancer associated weight loss indicated poor prognosis, thus higher BMI of patients with malignancy and slower weight loss during treatment suggests better survival rate [17]. The linkage of body weight and prognosis in patients receiving ICls therapy was first proved in our study. In subgroup of PD-1 inhibitors, the comorbidity of ICI-related thyroid dysfunction in ICI-PAI patients revealed a positive correlation with severe clinical outcomes, while no association was discovered between ICI-TD and the risk of death. A prospective study and an observational study indicated that the overall survival was significantly longer in patients on ICls treatment after developed ICl-related thyroid dysfunction, and the prognosis was even better in patients with overt hypothyroidism [18-20]. One possible explanation was that patients who developed irAEs might also have preferable treatment response, while irAEs in itself could bring about poor clinical outcomes. Therefore, more attention should be paid for the monitor of irAEs, especially patients with lowered body mass during ICls treatment.

No case of adrenal crisis was reported FAERS. A life-threatening disease as it is, less is known about IClinduced adrenal crisis. Regular measurement of serum cortisol and adrenocorticotropin could be recommended for patients on ICls treatment presented with fatigue or nausea, in the order of early diagnosis [21].

As a retrospective study, the limitations are associated to referral bias and the lack of follow-up. Since a proportion of non-serious cases may be not reported in FAERS database, it could result in bias to the analysis of prognosis. The incomplete data of FAERS database and the fact that adrenocorticotropic hormone (ACTH) level was not available in FAERS, which is critical in the differential diagnosis of primary and secondary adrenal insufficiency, led to inevitable bias to the incidence of ICI-PAI. Although the reaction terms including secondary or pituitary adrenal insufficiency and hypophysitis were excluded from our study population, the data of FAERS reporting is voluntary from endocrinologists as well as 
oncologists and general practitioners, and it was unrealistic to the confirmed diagnosis of PAI or central adrenal insufficiency for all reporters. In addition, our study focused on ICls-mediated new-diagnosed PAI instead of other causes of PAI including bilateral adrenal metastasis or hemorrhage, which brought about bias to data analysis.

\section{Conclusions}

ICl-associated PAI is a rare but important irAE. Male and elder patients have higher risk of ICI-PAI. The combined regimen of ipilimumab and nivolumab results in a higher possibility of polyendocrinopathies including PAI. Awareness among health care professionals is critical when patients with lower body weight develop PAl, which indicates poor clinical outcomes.

\section{Abbreviations}

\begin{tabular}{ll} 
Immune checkpoint inhibitor & ICl \\
\hline Programmed cell death protein-1 & PD-1 \\
\hline Programmed death-ligand 1 & PD-L1 \\
\hline Cytotoxic T cell-associated protein-4 & CTLA-4 \\
\hline Immune-related adverse events & irAEs \\
\hline Primary adrenal insufficiency & PAI \\
\hline FDA Adverse Event Reporting System & FAERS \\
\hline Type 1 diabetes mellitus & T1DM
\end{tabular}

\section{Declarations}

Ethics approval and consent to participate: All cases in the study were anonymous and all data was available in public resource, and this study was waived from informed consent and ethics.

Consent for publication: Not applicable.

Availability of data and materials: The datasets used and/or analysed during the current study are available from the corresponding author on reasonable request.

Competing interests: The authors declare that they have no competing interests.

Funding: The study was granted by Scientific Research Seed Fund of Peking University First Hospital (2018SF028). 
Authors' contributions: YG initiated the study and revised the manuscript. DL performed statistical analysis and wrote the manuscript. JY, GY, JZ and XG participated in manuscript revision.

Acknowledgement: Not applicable.

\section{References}

1. Brahmer JR, Lacchetti C, Schneider BJ, et al. National Comprehensive Cancer Network Management of immune-related adverse events in patients treated with immune checkpoint inhibitor therapy: American Society of Clinical Oncology Clinical Practice Guideline. J Clin Oncol. 2018; 36 (17): 17141768.

2. Barroso-Sousa R, Barry WT, Garrido-Castro AC, et al. Incidence of endocrine dysfunction following the use of different immune checkpoint inhibitor regimens: a systematic review and meta-analysis. JAMA Oncol. 2018; 4(2): 173-182.

3. Bancos I, Hahner S, Tomlinson J, et al. Diagnosis and management of adrenal insufficiency. Lancet Diabetes Endocrinol. 2015; 3(3): 216-226.

4. Byun DJ, Wolchok JD, Rosenberg LM, et al. Cancer immunotherapy-immune checkpoint blockade and associated endocrinopathies. Nat Rev Endocrinol. 2017; 13(4): 195-207.

5. Yang JC, Hughes M, Kammula Y, et al. Ipilimumab (anti-CTLA4 antibody) causes regression of metastatic renal cell cancer associated with enteritis and hypophysitis. J Immunother. 2007; 30(8): 825-830.

6. Traner $\mathrm{H}$, Hulse $\mathrm{P}$, Higham $\mathrm{CE}$, et al. Hyponatremia secondary to nivolumab-induced primary adrenal failure. Endocrinol Diabetes Metab Case Rep. 2016; 2016.

7. Min L, Ibrahim N. Ipilimumab-induced autoimmune adrenalitis. Lancet Diabetes Endocrinol. 2013; 1(3): e15.

8. Cukier P, Santini FC, Scaranti M, et al. Endocrine side effects of cancer immunotherapy. Endocr Relat Cancer. 2017; 24(12): T331-T347.

9. Lu J, Li L, Lan Y, et al. Immune checkpoint inhibitor-associated pituitary-adrenal dysfunction: a systematic review and meta-analysis. Cancer Medicine. 2019; 8: 7503-7515.

10. Grouthier V, Lebrun-Vignes B, Moey M, et al. Immune checkpoint inhibitor-associated primary adrenal insufficiency: WHO VigiBase report analysis. The Oncologists. 2020; 25: 696-701.

11. Triggianese P, Novelli L, Galdiero MR, et al. Immune checkpoint inhibitors-induced autoimmunity: the impact of gender. Autoimmun Rev. 2020; 19(8): 102590.

12. Zhai Y, Ye X, Hu F, et al. Endocrine toxicity of immune checkpoint inhibitors: a real-world study leveraging US Food and Drug Administration adverse events reporting system. Journal for Immuno Therapy of Cancer. 2019; 7:286.

13. Xu C, Chen YP, Du XJ, et al. Comparative safety of immune checkpoint inhibitors in cancer: systematic review and network meta-analysis. BMJ. 2018; 363: k4226. 
14. Sun X, Roudi R, Dai T, et al. Immune-related adverse events associated with programmed cell death protein-1 and programmed cell death ligand 1 inhibitors for non-small cell lung cancer: a PRISMA systematic review and meta-analysis. BMC Cancer. 2019; 19(1): 558.

15. Wolchok JD, Chiarion-Sileni C, Gonzalez R, et al. Overall survival with combined nivolumab and ipilimumab in advanced melanoma. N Engl J Med. 2017; 377(14): 1345-1356.

16. Arnaud-Coffin P, Maillet D, Gan HK, et al. A systematic review of adverse events in randomized trials assessing immune checkpoint inhibitors. Int J Cancer. 2019; 145(3): 639-648.

17. Martin L, Senesse P, Gioulbasanis I, et al. Diagnostic criteria for the classification of cancerassociated weight loss. J Clin Oncol. 2015; 33(1): 90-99.

18. Osorio JC, Ni A, Chaft JE, et al. Antibody-mediated thyroid dysfunction during T-cell checkpoint blockade in patients with non-small-cell lung cancer. Annals of Oncology. 2017; 28: 583-589.

19. Kim HI, Kim M, Lee SH, et al. Development of thyroid dysfunction is associated with clinical response to PD-1 blockade treatment in patients with advanced non-small cell lung cancer. Onco Immunology. 2017; 19: e1375642.

20. Yamauchi I, Yasoda A, Matsumoto S, et al. Incidence, features, and prognosis of immune-related adverse events involving the thyroid gland induced by nivolumab. PloS One. 2019; 14: e0216954.

21. Reznik $Y$, Barat $P$, Bertherat $J$, et al. SFE/SFEDP adrenal insufficiency French consensus: introduction and handbook. Ann Endocrinol (Paris). 2018; 79: 1-22. 